Ener gy- Ef fi ci ent Resour ce Al I ocat i on for D2D Communi cat i ons Under I ayi ng $\mathrm{Cl}$ oud- RAN Based LTE- A Net wor ks

\begin{tabular}{|l|l|}
\hline 著者 & $\begin{array}{l}\text { ZHOU Zhenyu, DONG M anxi ong, OTA Kaor u, WANG } \\
\text { Guoj un, YANG Laur ence T. }\end{array}$ \\
\hline $\begin{array}{l}\text { j our nal or } \\
\text { publ i cat i on ti t l e }\end{array}$ & I EEE I nt er net of Thi ngs J our nal \\
\hline vol une & 3 \\
\hline number & 3 \\
\hline page range & $428-438$ \\
\hline year & $2016-06$ \\
\hline URL & ht t p: //hdl . handl e. net /10258/00008998 \\
\hline
\end{tabular}




\title{
Energy-Efficient Resource Allocation for D2D Communications Underlaying Cloud-RAN based LTE-A Networks
}

\author{
Zhenyu Zhou, Member, IEEE, Mianxiong Dong, Member, IEEE, Kaoru Ota, Member, IEEE, \\ Guojun Wang, Member, IEEE, and Laurence T. Yang, Member, IEEE
}

\begin{abstract}
Device-to-Device (D2D) communication is a key enabler to facilitate the realization of the internet of things (IoT). In this paper, we study the deployment of D2D communications as an underlay to long term evolution-advanced (LTE-A) networks based on novel architectures such as cloud radio access network (C-RAN). The challenge is that both energy efficiency (EE) and quality of service (QoS) are severely degraded by the strong intracell and intercell interference due to dense deployment and spectrum reuse. To tackle this problem, we propose an energyefficient resource allocation algorithm through joint channel selection and power allocation design. The proposed algorithm has a hybrid structure that exploits the hybrid architecture of C-RAN: distributed remote radio heads (RRHs) and centralized baseband unit (BBU) pool. The distributed resource allocation problem is modeled as a noncooperative game, and each player optimizes its EE individually with the aid of distributed RRHs. We transform the non-convex optimization problem into a convex one by applying constraint relaxation and nonlinear fractional programming. We propose a centralized interference mitigation algorithm to improve the QoS performance. The centralized algorithm consists of an interference cancellation technique and a transmission power constraint optimization technique, both of which are carried out in the centralized BBU pool. The achievable performance of the proposed algorithm is analyzed through simulations, and the implementation issues and complexity analysis
\end{abstract}

Manuscript received April 15, 2015; revised October 6, 2015; accepted October 18, 2015

This work was partially supported by National Science Foundation of China (NSFC) under Grant Number 61203100, 71061005, JSPS KAKENHI Grant Number 26730056, 15K15976, and JSPS A3 Foresight Program, and Fundamental Research Funds for the Central Universities under Grant Number 13MS19, 14MS08, 15MS04, and China Electric Power Research Institute (CEPRI) of State Grid Corporation of China (SGCC).

Z. Zhou is with the State Key Laboratory of Alternate Electrical Power System with Renewable Energy Sources, School of Electrical and Electronic Engineering, North China Electric Power University, Beijing, China, 102206. E-mal: zhenyu_zhou@ncepu.edu.cn

M. Dong (corresponding author) and K. Ota are with the Department of Information and Electric Engineering, Muroran Institute of Technology, Muroran, Hokkaido, Japan. E-mail: mx.dong@csse.muroran-it.ac.jp, ota@csse.muroran-it.ac.jp

G. Wang is with School of Computer Science and Educational Software, Guangzhou University, Guangzhou 510006, China and School of Information Science and Engineering, Central South University, Changsha 410083, China. E-mal: csgjwang@gmail.com

L. T. Yang is with the Department of Mathematics, Statistics, and Computer Science, St. Francis Xavier University, Antigonish, NS B2G2W5, Canada. Emal: 1tyang@stfx.ca

Copyright (c) 2012 IEEE. Personal use of this material is permitted. However, permission to use this material for any other purposes must be obtained from the IEEE by sending a request to pubs-permissions@ieee.org are discussed in details.

Keywords-Internet of things, LTE-A, C-RAN, D2D, energy efficiency, resource allocation.

\section{INTRODUCTION}

$\mathbf{W}$ ITH the explosive growth of mobile Internet, it is predicted that mobile data traffic will grow more than 1000 times from 2010 to 2020, and almost 50 billion devices will be connected by then [1], [2]. Internet of things (IoT) that enables ubiquitous information acquisition and exchange among devices without external human intervention is a key enabler to facilitate the evolution of cellular network from long term evolution-advanced (LTE-A) systems to future 5G [?], [?].

Among various types of communication technologies existing within the IoT, device-to-device (D2D) communications allow user equipments (UEs) that are in the proximity of each other to exchange information over a direct link, and can be operated as an underlay to LTE-A networks by reusing the scarce spectrum resources [3]. D2D communications also enable the cooperation between UEs and machine-type devices (MTDs) [4], and function as a machine-type gateway (MTG) through data aggregation and relaying to reduce the number of connections between MTDs and base stations (BSs) [5]. The applications of D2D communications underlaying LTE-A networks were studied in [6], [7], and up-to-date standardization activities in 3rd generation partnership project (3GPP) were introduced in [8]. Furthermore, cloud radio access network (CRAN) based LTE-A systems are proposed to facilitate crosscell cooperations and improve spectrum efficiency (SE) by employing densely deployed remote radio heads (RRHs) that are powered by cloud computing to cooperatively support UEs [9], [10]. As a result, D2D communications underlaying CRAN will bring numerous benefits [11], and provide flexible control through centralized network coordination and advanced resource allocation techniques [12]. First of all, unlike other IoT based applications, D2D communications require much higher throughput and QoS. Thus, better performance can be achieved for D2D communications with the assistance and support of the densely deployed RRHs and centralized baseband unit (BBU) pool because the computational performance of UEs is far lower than that of C-RAN. Second, C-RAN has the disadvantages of severe intercell interference and fronthaul 
capacity constraints. The integration offers advantages of shifting the data transmission load from a big cloud to UEs and reducing the latency and volume of data signaling between the BBU pool and end UEs. Last but not least, it shed lights on the convergence of C-RAN based LTE-A networks with D2D underlay that enables the coexistence of different types of communication protocols, services, and devices under a unified frame.

However, the integration of D2D with C-RAN based LTE-A networks poses many new challenges in the resource allocation design due to the dense deployment of RRHs and intense spectrum reuse. Both the energy efficiency (EE) and quality of service (QoS) are challenged by the strong intracell and intercell interference. For example, UEs near the edge of a cell have to increase their transmission power to meet the QoS requirement. As a result, UEs with limited battery capacity will quickly run out of battery if without careful energy optimization and interference management design.

In this paper, we propose an energy-efficient resource allocation algorithm to optimize EE while guaranteeing the QoS provisioning through joint channel selection and power allocation design. The proposed algorithm has a hybrid structure that exploits the hybrid architecture of C-RAN: distributed RRHs and centralized BBU pool. Firstly, the available resources are scheduled by each UE in an energy-efficient way. Since UEs are only interested in improving their own performance, we propose a game-theoretic approach which models the distributed resource allocation problem as a noncooperative game. Each player optimizes its EE individually with the aid of distributed RRHs under the transmission power and QoS constraints. We transform the non-convex optimization problem into a convex one by applying constraint relaxation and nonlinear fractional programming. We prove that a Nash equilibrium exists in the noncooperative game, and the optimum solution obtained by the proposed algorithm is exactly the Nash equilibrium. Secondly, we propose a centralized interference mitigation algorithm to improve the QoS performance. The strong intercell interference caused by cellular UEs is canceled by exchanging decoded dominant interferer data in the centralized BBU pool and reconstructing interfering signals. The interference caused by D2D UEs is also mitigated by dynamically optimizing maximum transmission power constraints in the BBU pool. Finally, the achievable performance of the proposed algorithm and the impacts of channel estimation errors are analyzed through simulations, and the implementation and complexity issues are discussed in details.

The structure of this paper is organized as follows: Section II describes the related works. Section III introduces the system model of D2D communications underlaying C-RAN. Section IV introduces the problem formulation. Section $\mathrm{V}$ introduces the proposed hybrid energy-efficient resource allocation algorithm. Section VI introduces the simulation parameters, results and analyses. Section VII gives the conclusion.

\section{RELATED WORKS}

Game theory provides a tool set for analyzing optimization problems with multiple conflicting objective functions and has been widely used for resource allocation in D2D communications. A Stackelberg game based resource allocation scheme was proposed in [13], in which the Base Station (BS) and D2D UEs were modeled as the game leader and followers respectively. Another Stackelberg game based scheme was proposed in [14], in which cellular UEs rather than the BS were modeled as game leaders. A two-stage resource allocation scheme which employs both the centralized and distributed approaches was proposed in [15]. A three-stage resource allocation scheme which combines admission control, power allocation, and link selection was proposed in [16]. A reverse Iterative Combinatorial Auction (ICA) based resource allocation scheme was proposed in [17] for optimizing the system sum rate. The resource allocation problems in relay-aided scenarios were studied in [18], [19], and in infeasible systems where all users can not be supported simultaneously were studied in [20]. The throughput performance of the D2D underlay communications with different resource sharing modes was evaluated in [21]. SE enhancement of D2D communications for wireless video networks was studied in [22]. Resource allocation for D2D communications underlaying cellular networks powered by renewable energy sources was studied in [23]. A comprehensive overview and discussion of resource management for D2D underlay communications is provided in [24].

The above mentioned works mainly focus on how to maximize SE and ignore the energy consumption of UEs. A few works have addressed the energy-efficient resource allocation problem in the conventional single-cell scenario. Centralized resource allocation algorithms for optimizing EE in the deviceto-multi-device (D2MD) or D2D-cluster scenarios were studied in [25] and [26] respectively. An auction-based energyefficient resource allocation algorithm was proposed in [27], and auction-based resource allocation scheme and D2D cooperative relays were proposed to improve battery lifetime in [28] and [29] respectively. Fractional frequency reuse (FFR) based two-stage resource allocation algorithm was proposed in [30]. An iterative resource allocation scheme was proposed in [31], and the EE-SE tradeoff was studied in [32]. Coalition game based resource allocation algorithms were proposed to jointly optimize the model selection and resource scheduling in [33], [34].

However, the above works lack the detailed modeling of complex interference scenarios in densely deployed multicellular networks, and are not designed based on the novel architecture of C-RAN. Furthermore, they focus either on centralized or distributed approaches, and have not taken advantages of both of them. The conventional centralized resource allocation problem is not convex due to the coupling of the resource allocation strategies. As a result, the centralized problem is NP-hard, and it is intractable to find the global optimum solution of the overall network. In addition, the computational complexity and signaling overhead increase significantly with the number of UEs, and the optimum solution needs to be delivered from the BBU pool to UEs within the channel coherence time. In comparison, the proposed hybrid scheme provides a flexible tradeoff between performance improvements and implementation complexity, since the centralized interference cancellation and mitigation algorithm is invoked 
only if the intercell interference exceeds a certain threshold. It also enables seamless coexistence with the heterogeneous architecture of D2D communications underlaying C-RAN based LTE-A networks. Better performance can be achieved for D2D communications with the assistance and support of the densely deployed RRHs and centralized BBU pool. At the same time, the data load can be shifted from constrained fronthaul links to UEs to further reduce transmission latency and signaling overhead.

\section{SYSTEM MODEL}

The general architecture of D2D communications underlaying C-RAN is shown in Fig. 1, which consists of the following main components: (i) a BBU pool, (ii) RRHs, (iii) fronthaul links, (iv) cellular UEs, and (v) D2D UEs. We focus on the uplink resource sharing scenario where D2D UEs are allowed to reuse the uplink spectrum resources allocated to cellular UEs. RRHs are responsible for forwarding baseband signals from cellular UEs to the BBU pool for further processing in the uplink [1]. In general, RRHs are configured with simple front radio frequency $(\mathrm{RF})$ and signal processing functionalities such as RF amplification, up/down conversion, filtering, A/D and D/A conversion, etc. The BBU pool consists of BBUs which can be implemented in the form of virtual base stations (VBS) to enable network function virtualization (NFV) [35]. The $\mathrm{BBU}$ pool is configured with powerful centralized processors to process baseband signals and optimize resource allocation. The fronthaul links connect the RRHs with the BBU pool and require low latency. The fronthaul links can be realized by different technologies [1].

Fig. 2 shows an example of the complex interference environment of two active cells. There are two cellular UEs (UE ${ }_{1}$ and $\left.\mathrm{UE}_{2}\right)$, and two D2D pairs $\left(\mathrm{UE}_{3}\right.$ and $\mathrm{UE}_{4}$, and $\mathrm{UE}_{5}$ and $\mathrm{UE}_{6}$ respectively). Since each cellular UE is allocated with an orthogonal link (e.g., an orthogonal resource block in LTE-A), i.e., there is no intracell interference among cellular UEs in the same cell, but there is intercell interference among cellular UEs located in adjacent cells. Block fading model where the channel gain is constant during a slot is adopted [36]. As a result, the $\mathrm{RRH}_{1}$ suffers from the intercell interference caused by the cellular UE in the adjacent cell $\left(\mathrm{UE}_{2}\right)$ as shown in Fig. 2(a), the intracell interference caused by the D2D transmitter in the same cell $\left(\mathrm{UE}_{3}\right)$ as shown in Fig. 2(b), and the intercell interference caused by the D2D transmitter in the adjacent cell $\left(\mathrm{UE}_{5}\right)$ as shown in Fig. 2(c). D2D receivers $\left(\mathrm{UE}_{4}\right.$ and $\left.\mathrm{UE}_{6}\right)$ suffer from the interference caused by cellular UEs (UE $\mathrm{UE}_{1}$ and $\mathrm{UE}_{2}$ ) as shown in Fig. 2(b), (c), and D2D transmitters that reuse the same channel ( $\mathrm{UE}_{5}$ or $\mathrm{UE}_{3}$ respectively) as shown in Fig. 2(d). As the density of active RRHs increases, it will cause severe interference that leads to inefficient $\mathrm{EE}$ and degraded QoS.

For a more general case, we consider a total of $M(M \geq 2)$ adjacent cells. In the $m$-th cell $(m=1, \cdots, M)$, the set of UEs is denoted as $\mathcal{S}_{m}=\left\{\mathcal{N}_{m}, \mathcal{K}_{m}\right\}$, where $\mathcal{N}_{m}$ and $\mathcal{K}_{m}$ denote the sets of D2D UEs and cellular UEs respectively. The total numbers of D2D links and cellular links are denoted as $N_{m}$ and $K_{m}$ respectively. The achievable SE (defined as bits $/ \mathrm{s} / \mathrm{Hz}$ )

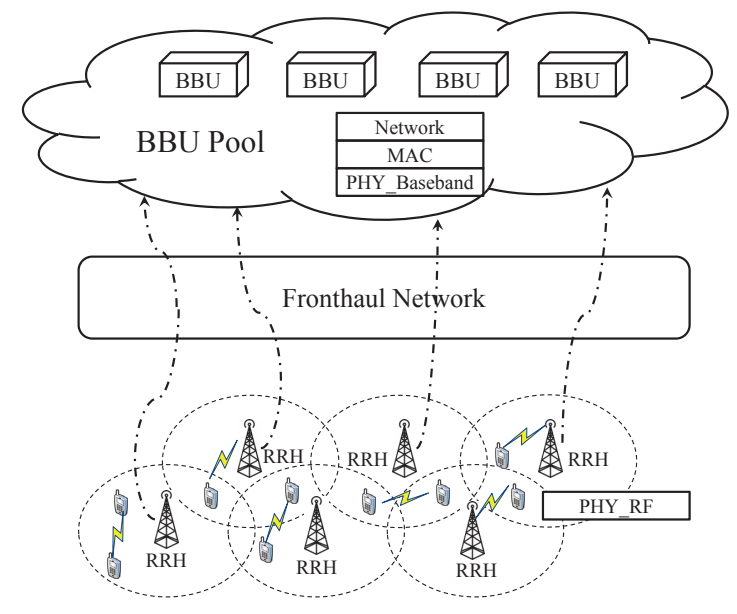

Fig. 1. The architecture of D2D communications underlaying C-RAN.

of the $i$-th D2D pair in the $m$-th cell $\left(i \in \mathcal{N}_{m}, m \in \mathcal{M}\right)$ on the $k$-th channel $\left(k \in \mathcal{K}_{m}\right)$ is given by

$$
C_{i_{m}}^{d}=\sum_{k \in K_{m}} \log _{2}\left(1+\frac{s_{i_{m}}^{k} p_{i_{m}}^{k} g_{i_{m}}^{k}}{I_{i_{m}, 1}^{d}+I_{i_{m}, 2}^{d}+I_{i_{m}, 1}^{c}+I_{i_{m}, 2}^{c}+N_{0}}\right),
$$

where $p_{i_{m}}^{k}$ is the transmission power of the $i$-th D2D transmitter on the $k$-th channel in the $m$-th cell. $g_{i_{m}}^{k}$ is the desired D2D signal channel gain. $s_{i_{m}}^{k}$ is the binary channel selection indicator, i.e., $s_{i_{m}}^{k}=1$ means that the $k$-th channel is reused by the $i$-th D2D UE of the $m$-th cell, and otherwise, $s_{i_{m}}^{k}=0$. $N_{0}$ is the noise power.

$I_{i_{m}, 1}^{d}$ and $I_{i_{m}, 2}^{d}$ denote the intracell interference and intercell interference caused by D2D UEs, and are given by

$$
\begin{aligned}
I_{i_{m}, 1}^{d} & =\sum_{j_{m} \in \mathcal{N}_{m} \backslash\left\{i_{m}\right\}} s_{j_{m}}^{k} p_{j_{m}}^{k} g_{j_{m}, i_{m}}^{k}, \\
I_{i_{m}, 2}^{d} & =\sum_{m^{\prime} \in \mathcal{M} \backslash\{m\}} \sum_{i_{m^{\prime}} \in \mathcal{N}_{m^{\prime}}} s_{i_{m^{\prime}}}^{k} p_{i_{m^{\prime}}}^{k} g_{i_{m^{\prime}}, i_{m}}^{k},
\end{aligned}
$$

where $s_{j_{m}}^{k} p_{j_{m}}^{k} g_{j_{m}, i_{m}}^{k}$ is the intracell interference from the $j$-th D2D interferer to the $i$-th D2D receiver on the $k$-th channel, i.e., $j \neq i . s_{i_{m^{\prime}}}^{k} p_{i_{m^{\prime}}}^{k}, g_{i_{m^{\prime}}, i_{m}}^{k}$ is the intercell interference on the $k$-th channel from the $i$-th D2D interferer in the $m^{\prime}$-th cell to the $i$-th D2D receiver in the $m$-th cell, i.e., $m^{\prime}=1,2, \cdots, M$, $m^{\prime} \neq m$.

$I_{i_{m}, 1}^{c}$ and $I_{i_{m}, 2}^{c}$ denote the intracell interference and intercell interference caused by cellular UEs, and are given by

$$
\begin{aligned}
I_{i_{m}, 1}^{c} & =p_{k_{m}}^{c} g_{k_{m}, i_{m}}^{k}, \\
I_{i_{m}, 2}^{c} & =\sum_{m^{\prime} \in \mathcal{M} \backslash\{m\}} p_{k_{m^{\prime}}}^{c} g_{k_{m^{\prime}}, i_{m}}^{k},
\end{aligned}
$$

where $p_{k_{m}}^{c} g_{k_{m}, i_{m}}^{k}$ denotes the intracell interference from the $k$-th cellular interferer to the $i$-th D2D receiver. $p_{k_{m^{\prime}}}^{c} g_{k_{m^{\prime}}, i_{m}}^{k}$ 


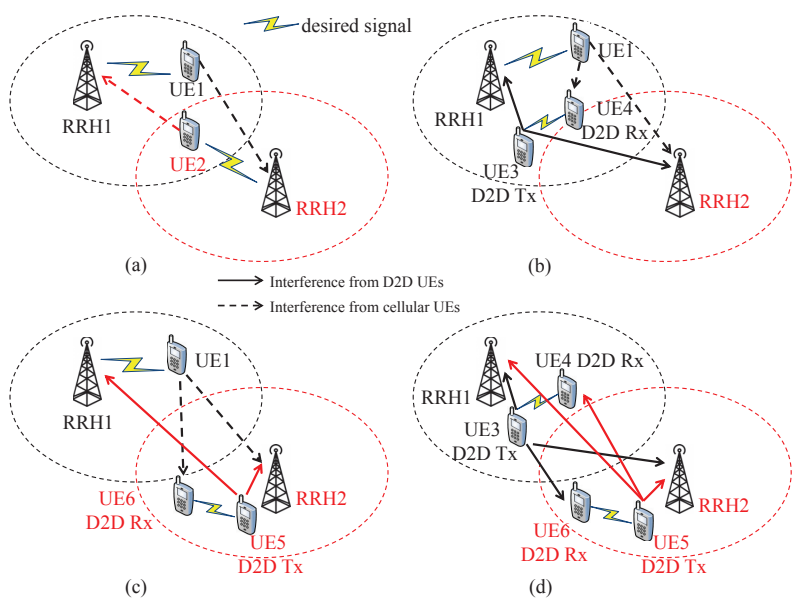

Fig. 2. An example of D2D communications underlaying C-RAN with two cells: (a). intercell interference among cellular UEs; (b). intracell interference among cellular and D2D UEs; (c). intercell interference among cellular and D2D UEs; (d). intercell interference among D2D UEs.

denotes the intercell interference from the $k$-th cellular interferer in the $m$-th cell to the $i$-th D2D receiver in the $m$-th cell.

Similarly, the SE of the $k$-th cellular UE in the $m$-th cell is given by

$$
C_{k_{m}}^{c}=\log _{2}\left(1+\frac{p_{k_{m}}^{c} g_{k_{m}}^{c}}{I_{k_{m}, 1}^{d}+I_{k_{m}, 2}^{d}+I_{k_{m}, 2}^{c}+N_{0}}\right),
$$

where $p_{k_{m}}^{c} g_{k_{m}}^{c}$ is the desired signal received at the associated RRH. $I_{k_{m}, 1}^{d}$ denotes the intracell interference caused by D2D UEs in the same cell, and is given by

$$
I_{k_{m}, 1}^{d}=\sum_{i \in \mathcal{N}_{m}} s_{i_{m}}^{k} p_{i_{m}}^{k} g_{i_{m}, k_{m}}^{c}
$$

where $s_{i_{m}}^{k} p_{i_{m}}^{k} g_{i_{m}, k_{m}}^{c}$ is the intracell interference from the $i$-th D2D interferer on the $k$-th channel in the $m$-th cell.

$I_{k_{m}, 2}^{d}$ denotes the intercell interference caused by D2D UEs in adjacent cells, and is given by

$$
I_{k_{m}, 2}^{d}=\sum_{m^{\prime} \in \mathcal{M} \backslash\{m\}} \sum_{i_{m^{\prime}} \in \mathcal{N}_{m^{\prime}}} s_{i_{m^{\prime}}}^{k} p_{i_{m^{\prime}}}^{k} g_{j_{m^{\prime}}, k_{m}}^{c},
$$

where $s_{i_{m^{\prime}}}^{k} p_{i_{m^{\prime}}}^{k} g_{j_{m^{\prime}}, k_{m}}^{c}$ is the intercell interference on the $k$-th channel from the $i$-th D2D interferer in the $m^{\prime}$-th cell.

$I_{k_{m}, 2}^{c}$ denotes the intercell interference caused by cellular UEs in adjacent cells, and is given by

$$
I_{k_{m}, 2}^{c}=\sum_{m^{\prime} \in \mathcal{M} \backslash\{m\}} p_{k_{m^{\prime}}}^{c} g_{k_{m^{\prime}}, k_{m}}^{c},
$$

\begin{tabular}{|c|c|}
\hline Parameter & Description \\
\hline $\mathcal{M}$ & the set of cells \\
\hline $\mathcal{K}_{m}$ & the set of cellular UEs in the $m$-th cell \\
\hline $\mathcal{N}_{m}$ & the set of D2D pairs in the $m$-th cell \\
\hline$i_{m}, k_{m}$ & $\begin{array}{l}\text { the indexes of the } i \text {-th D2D pair, and the } k \text {-th } \\
\text { cellular UE in the } m \text {-th cell, respectively }\end{array}$ \\
\hline$C_{i_{m}}^{d}$ & SE of the $i$-th D2D pair in the $m$-th cell \\
\hline$C_{k_{m}}^{c}$ & SE of the $k$-th cellular UE in the $m$-th cell \\
\hline$E_{i_{m}}^{d m}$ & EE of the $i$-th D2D pair in the $m$-th cell \\
\hline$E_{k m}^{c}$ & EE of the $k$-th cellular UE in the $m$-th cell \\
\hline$p_{i_{m}, t}^{d}, p_{k_{m}, t}^{c}$ & total power consumption \\
\hline$\left\{\mathbf{s}_{i_{m}}^{d}, \mathbf{p}_{i_{m}}^{d}\right\}$ & $\begin{array}{l}\text { the channel selection and transmission power } \\
\text { strategy set of the } i \text {-th D2D pair in the } m \text {-th cell }\end{array}$ \\
\hline$\left\{\mathbf{s}_{i_{m}}^{d *}, \mathbf{p}_{i_{m}}^{d *}, \mathbf{p}_{k_{m}}^{c *}\right\}$ & the optimum resource allocation strategy set \\
\hline $\mathbf{p}_{k_{m}}^{c}$ & $\begin{array}{l}\text { the transmission power strategy set of the } \\
k \text {-th cellular UE in the } m \text {-th cell }\end{array}$ \\
\hline$s_{i_{m}}^{k}$ & $\begin{array}{l}\text { the } i \text {-th D2D pair's channel selection indicator } \\
\text { for the } k \text {-th channel }\end{array}$ \\
\hline$p_{i_{m}}^{k}$ & $\begin{array}{l}\text { the } i \text {-th D2D pair's transmission power } \\
\text { on the } k \text {-th channel }\end{array}$ \\
\hline$p_{k_{m}}^{c}$ & $\begin{array}{l}\text { the } k \text {-th cellular UE's transmission } \\
\text { power on the } k \text {-th channel }\end{array}$ \\
\hline$p_{i_{m}, \max }^{d}, p_{k_{\operatorname{m}, \max }}^{c}$ & maximum transmission power constraints \\
\hline$p_{i_{m}, \max }^{k}, w_{i_{m, \max }}^{k}$ & $\begin{array}{l}\text { the maximum transmission power constraint } \\
\text { on the } k \text {-th channel and its corresponding weight }\end{array}$ \\
\hline$p_{\text {cir }}$ & UE's circuit power consumption \\
\hline$\eta$ & power amplifier efficiency \\
\hline$C_{k_{m}, \min }^{c}$ & QoS requirement \\
\hline$q_{i_{m}}^{d *}, q_{k_{m}}^{c *}$ & maximum achievable EE \\
\hline
\end{tabular}

where $p_{k_{m^{\prime}}}^{c} g_{k_{m^{\prime}}, k_{m}}^{c}$ is the intercell interference from the $k$-th cellular interferer in the $m^{\prime}$-th cell.
TABLE I. PARAMETER NOTATIONS.

The total power consumptions are given by

$$
\begin{aligned}
& p_{i_{m}, t}^{d}=\sum_{k \in \mathcal{K}_{m}} \frac{1}{\eta} s_{i_{m}}^{k} p_{i_{m}}^{k}+2 p_{c i r} \\
& p_{k_{m}, t}^{c}=\frac{1}{\eta} p_{k_{m}}^{c}+p_{c i r}
\end{aligned}
$$

where $p_{i_{m}, t}^{d}$ is the total power consumption of the $i$-th D2D pair, which is composed of the transmission power over all of the $K_{m}$ channels, i.e., $\sum_{k \in \mathcal{K}_{m}} \frac{1}{\eta} s_{i_{m}}^{k} p_{i_{m}}^{k}$, and the circuit power of both the D2D transmitter and receiver, i.e., $2 p_{\text {cir }}$. The circuit power of any UE is assumed as the same and is denoted as $p_{c i r}$. $\eta$ is the power amplifier (PA) efficiency, i.e., $0<\eta<1 . p_{k_{m}, t}^{c}$ is the total power consumption of the $k$-th cellular UE, which is composed of the transmission power $(1 / \eta) p_{k_{m}}^{c}$ and the circuit power only at the transmitter side. The power consumption of RRHs is not taken into consideration since RRHs are usually powered by external grid power.

To improve the clarity, notations of key parameters are summarized in Table I.

\section{PRoblem Formulation}

In the distributed resource allocation scenario, UEs are assumed as rational and selfish [37], i.e., each UE is interested in maximizing its individual utility through resource allocation. Therefore, the distributed power allocation problem can be modeled as a noncooperative game $\mathcal{G}$. The game $\mathcal{G}$ can be described as the triplet $\mathcal{G}=[\{\mathcal{S}\},\{\mathcal{A}\},\{\mathcal{U}\}]$, wherein $\mathcal{S}=$ $\left\{\mathcal{S}_{1}, \mathcal{S}_{2}, \cdots, \mathcal{S}_{M}\right\}$ is the set of active UEs participating in the 
game, $\mathcal{A}=\left\{\mathcal{A}_{1}, \mathcal{A}_{2}, \cdots, \mathcal{A}_{M}\right\}$ is the set of possible actions (strategies) that UEs can take, and $\mathcal{U}=\left\{\mathcal{U}_{1}, \mathcal{U}_{2}, \cdots, \mathcal{U}_{M}\right\}$ is the set of UEs' utilities. As an example, if $\mathcal{A}_{m}=\left\{p_{\max }\right\}$, then each UE in the $m$-th cell is allowed to transmit power from the interval $\left[0, p_{\max }\right]$, with $p_{\max }$ as the maximum allowed transmission power.

To be more general, the transmission power and channel selection strategy set of the $i$-th D2D transmitter in the $m$-th cell is denoted as $\left\{\mathbf{s}_{i_{m}}^{d}, \mathbf{p}_{i_{m}}^{d}\right\}=\left\{s_{i_{m}}^{k}, p_{i_{m}}^{k} \mid s_{i_{m}}^{k}=\{0,1\}, 0 \leq\right.$ $\left.\sum_{k \in \mathcal{K}_{m}} s_{i_{m}}^{k} p_{i_{m}}^{k} \leq p_{i_{m}, \max }^{d^{n}}\right\}, \forall i \in \mathcal{N}_{m}, \forall k \in \mathcal{K}_{m}, \forall m \in \mathcal{M}$. The transmission power strategy set of $k$-th cellular UE in the $m$-th cell is denoted as $\mathbf{p}_{k_{m}}^{c}=\left\{p_{k_{m}}^{c} \mid 0 \leq p_{k_{m}}^{c} \leq p_{k_{m}, \max }^{c}\right\}$, $\forall k \in \mathcal{K}_{m}, \forall m \in \mathcal{M} . p_{i_{m}, \max }^{d}$ and $p_{k_{m}, \max }^{c}$ are the maximum transmission power constraints. The strategy sets of the other D2D transmitters and the other cellular UEs are denoted as $\left\{\mathbf{s}_{-i_{m}}^{d}, \mathbf{p}_{-i_{m}}^{d}\right\}=\left\{s_{j_{m}}^{k}, p_{j_{m}}^{k} \mid s_{j_{m}}^{k}=\{0,1\}, 0 \leq\right.$ $\left.\sum_{k \in \mathcal{K}_{m}} s_{j_{m}}^{k} p_{j_{m}}^{k} \leq p_{j_{m}, \text { max }}^{d}, j_{m} \neq i_{m}\right\}$, and $\mathbf{p}_{-k_{m}}^{c}=\left\{p_{k_{m}}^{c}\right.$, $\left.0 \leq p_{k_{m^{\prime}}}^{c} \leq p_{k_{q^{\prime}, \max }}^{c}, m^{\prime} \neq m\right\}$ respectively, $\forall i \in \mathcal{N}_{m}$, $\forall k \in K_{m}^{m^{\prime}}, \forall m \in \mathcal{M}^{\prime}$.

The utility function is defined as the $\mathrm{EE}$ (bits/J/Hz), which is the ratio of transmission rate to power consumption [38]. The EE of the $i$-th D2D pair (including both the D2D transmitter and receiver) in the $m$-th cell is defined as

$$
E_{i_{m}}^{d}\left(\mathbf{s}_{i_{m}}^{d}, \mathbf{p}_{i_{m}}^{d}\right)=\frac{C_{i_{m}}^{d}\left(\mathbf{s}_{i_{m}}^{d}, \mathbf{p}_{i_{m}}^{d}\right)}{p_{i_{m}, t}^{d}\left(\mathbf{s}_{i_{m}}^{d}, \mathbf{p}_{i_{m}}^{d}\right)}
$$

The corresponding EE optimization problem is formulated as

$$
\begin{array}{lc}
\max _{\left(\mathbf{s}_{i_{m}}^{d}, \mathbf{p}_{i_{m}}^{d}\right)} & E_{i_{m}}^{d}\left(\mathbf{s}_{i_{m}}^{d}, \mathbf{p}_{i_{m}}^{d}\right) \\
\text { s.t. } & C_{1}: 0 \leq \sum_{k \in \mathcal{K}_{m}} s_{i_{m}}^{k} p_{i_{m}}^{k} \leq p_{i_{m}, \text { max }}^{d}, \\
& C_{2}: s_{i_{m}}^{k}=\{0,1\}, \forall k \in \mathcal{K}_{m} .
\end{array}
$$

$C_{1}$ is the maximum transmission power constraint, i.e., the total transmission power allocated over all of the $K_{m}$ channels should be no greater than $p_{i_{m}, \max }^{d} . C_{2}$ is the Boolean constraint of channel selection. Since D2D UEs communicate in a cognitive way, we have not imposed any QoS requirement for D2D UEs.

Similarly, the EE of the $k$-th cellular UE in the $m$-th cell, i.e., $E_{k_{m}}^{c}$, is defined as

$$
E_{k_{m}}^{c}\left(\mathbf{p}_{k_{m}}^{c}\right)=\frac{C_{k_{m}}^{c}\left(\mathbf{p}_{k_{m}}^{c}\right)}{p_{k_{m}, t}^{c}\left(\mathbf{p}_{k_{m}}^{c}\right)} .
$$

The corresponding EE maximization problem is formulated as

$$
\begin{array}{lc}
\underset{\left(\mathbf{p}_{k_{m}}^{c}\right)}{\max } & E_{k_{m}}^{c}\left(\mathbf{p}_{k_{m}}^{c}\right) \\
\text { s.t. } & C_{3}: C_{k_{m}}^{c}\left(\mathbf{p}_{k_{m}}^{c}\right) \leq C_{k_{m}, \min }^{c}, \\
& C_{4}: 0 \leq p_{k_{m}}^{c} \leq p_{k_{m}, \max }^{c} .
\end{array}
$$

The constraint $C_{3}$ specifies the QoS requirement in terms of minimum transmission rate, and the constraint $C_{4}$ specifies the transmission power constraint.

There are two challenges when solving the above optimization problems. Firstly, the problems are non-convex due to the fractional form and Boolean variables, and are computationally intractable. Secondly, the cellular UE's transmission rate defined in (6) depends on both intercell and intracell interference. If the combined interference is strong, the QoS constraint $C_{3}$ cannot be guaranteed and the problem (15) becomes infeasible.

\section{The EnERgy-EFFicient Resource Allocation ALGORITHM}

In this section, we propose an energy-efficient algorithm to address the above mentioned challenges. Firstly, we introduce a transformation to handle the non-convex problem via nonlinear fractional programming and constraint relaxation. Secondly, we propose an iterative algorithm (known as the Dinkelbach method) to solve the transformed problem. Thirdly, we analyze the Nash equilibrium of the noncooperative game, and its relationships with the optimum solution. Fourthly, we introduce a centralized interference mitigation scheme to improve the QoS performance. Finally, we discuss the implementation issues and provide complexity analysis.

\section{A. The Objective Function Transformation}

We start with the distributed resource allocation problem. Without loss of generality, the maximum EE of the $i$-th D2D pair in the $m$-th cell, $q_{i_{m}}^{d *}$, is defined as

$$
q_{i_{m}}^{d *}=\max _{\left(\mathbf{s}_{i_{m}}^{d}, \mathbf{p}_{i_{m}}^{d}\right)} E_{i_{m}}^{d}\left(\mathbf{s}_{i_{m}}^{d}, \mathbf{p}_{i_{m}}^{d}\right)=\frac{C_{i_{m}}^{d}\left(\mathbf{s}_{i_{m}}^{d *}, \mathbf{p}_{i_{m}}^{d *}\right)}{p_{i_{m}, t}^{d}\left(\mathbf{s}_{i_{m}}^{d *}, \mathbf{p}_{i_{m}}^{d *}\right)},
$$

where $\mathbf{s}_{i_{m}}^{d *}$ and $\mathbf{p}_{i_{m}}^{d *}$ are the optimum channel selection and power allocation strategies, respectively. The following theorem can be easily proved by following a similar approach as in [39]:

Theorem 1: $q_{i_{m}}^{d *}$ is achieved if and only if

$$
\begin{aligned}
& \max _{\left(\mathbf{s}_{i_{m}}^{d}, \mathbf{p}_{i_{m}}^{d}\right)} C_{i_{m}}^{d}\left(\mathbf{s}_{i_{m}}^{d}, \mathbf{p}_{i_{m}}^{d}\right)-q_{i_{m}}^{d *} p_{i_{m}, t}^{d}\left(\mathbf{s}_{i_{m}}^{d}, \mathbf{p}_{i_{m}}^{d}\right) \\
= & C_{i_{m}}^{d}\left(\mathbf{s}_{i_{m}}^{d *}, \mathbf{p}_{i_{m}}^{d *}\right)-q_{i_{m}}^{d *} p_{i_{m}, t}^{d}\left(\mathbf{s}_{i_{m}}^{d *}, \mathbf{p}_{i_{m}}^{d *}\right)=0 .
\end{aligned}
$$

Theorem 1 shows that the transformed problem with an objective function in subtractive form is equivalent to the non-convex problem with an objective function in fractional form. Therefore, we can focus on the equivalent transformed objective function, and the original problem (13) can be rewritten as

$$
\begin{array}{ll}
\max _{\left(\mathbf{s}_{i_{m}}^{d}, \mathbf{p}_{i_{m}}^{d}\right)} & C_{i_{m}}^{d}\left(\mathbf{s}_{i_{m}}^{d}, \mathbf{p}_{i_{m}}^{d}\right)-q_{i_{m}}^{d *} p_{i_{m}, t}^{d}\left(\mathbf{s}_{i_{m}}^{d}, \mathbf{p}_{i_{m}}^{d}\right), \\
\text { s.t. } & C_{1}, C_{2} .
\end{array}
$$

The new problem can be viewed as a weighted sum of SE and power consumption, where the parameter $q_{i_{m}}^{d *}$ acts as the "price" (negative weight) of the power consumption.

Although the objective function is now transformed into a subtractive form which is easier to handle, the equivalent problem (18) is still a mixed combinatorial and convex optimization problem. An exhaustive search is needed to obtain the optimum solution in every time instant, which leads to a complexity of $\mathcal{O}\left(N_{m}^{K_{m}}\right)$. In order to strike a balance between 
tractability and optimality, we can relax $s_{i}^{k}$ in constraint $C_{2}$ from a Boolean value to a real value between zero and one [40], i.e., $0 \leq s_{i_{m}}^{k} \leq 1$. Then, $s_{i_{m}}^{k}$ can be interpreted as a time sharing factor for $N_{m}$ users to utilize the $k$-th channel. The duality gap of the relaxation becomes negligible as the number of channels becomes sufficiently large [41]. Under this assumption, we can replace $s_{i_{m}}^{k} p_{i_{m}}^{k}$ by a new variable $\tilde{p}_{i_{m}}^{k}$.

Similarly, defining $q_{k_{m}}^{c *}$ as the maximum achievable EE of the $k$-th cellular UE in the $m$-th cell, the original problem (15) can be rewritten as

$$
\begin{array}{ll}
\underset{\left(\mathbf{p}_{k_{m}}^{c}\right)}{\max } & C_{k_{m}}^{c}\left(\mathbf{p}_{k_{m}}^{c}\right)-q_{k_{m}}^{c *} p_{k_{m}, t}^{c}\left(\mathbf{p}_{k_{m}}^{c}\right), \\
\text { s.t. } & C_{3}, C_{4} .
\end{array}
$$

However, the maximum $\mathrm{EE} q_{i_{m}}^{d *}$ and $q_{k_{m}}^{c *}$ are still unknown. In the next subsection, we introduce how to find $q_{i_{m}}^{d *}$ or $q_{k_{m}}^{c *}$ by proposing an iterative algorithm.

\section{B. The Iterative Resource Allocation Algorithm}

In this subsection, an iterative algorithm is proposed based on Dinkelbach's method [39] to find $q_{i_{m}}^{d *}$ or $q_{k_{m}}^{c *}$ for solving the transformed problems. The initial values of $q_{i_{m}}^{d}$ or $q_{k_{m}}^{c}$ can be set as a small positive number near to zero, e.g., $10^{-4}$. At each iteration, the following transformed problems are solved

$$
\begin{array}{ll}
\max _{\left(\tilde{\mathbf{p}}_{i_{m}}^{d}\right)} & C_{i_{m}}^{d}\left(\tilde{\mathbf{p}}_{i_{m}}^{d}\right)-q_{i_{m}}^{d} p_{i_{m}, t}^{d}\left(\tilde{\mathbf{p}}_{i_{m}}^{d}\right), \\
\text { s.t. } & C_{1}^{\prime}: 0 \leq \sum_{k \in \mathcal{K}_{m}} \tilde{p}_{i_{m}}^{k} \leq p_{i_{m}, \text { max }}^{d}, \\
& C_{2}^{\prime}: 0 \leq s_{i_{m}}^{k} \leq 1 . \\
\max _{\left(\mathbf{p}_{k_{m}}^{c}\right)} . & C_{k_{m}}^{c}\left(\mathbf{p}_{k_{m}}^{c}\right)-q_{k_{m}}^{c} p_{k_{m}, t}^{c}\left(\mathbf{p}_{k_{m}}^{c}\right), \\
\text { s.t. } & C_{3}, C_{4} .
\end{array}
$$

Since both (20) and (21) are convex, Karush-Kuhn-Tucker (KKT) conditions can be used to find the optimum strategy [42]. Taking (20) as an example, the associated Lagrangian is given by

$$
\begin{gathered}
\mathcal{L}_{E E}\left(\tilde{\mathbf{p}}_{i_{m}}^{d}, \alpha_{i_{m}}\right)=C_{i_{m}}^{d}\left(\tilde{\mathbf{p}}_{i_{m}}^{d}\right) \\
-q_{i_{m}}^{d} \tilde{p}_{i_{m}, t}^{d}\left(\tilde{\mathbf{p}}_{i_{m}}^{d}\right)-\alpha_{i_{m}}\left(\sum_{k \in \mathcal{K}_{m}} \tilde{p}_{i_{m}}^{k}-p_{i_{m}, \text { max }}^{d}\right),
\end{gathered}
$$

where $\alpha_{i_{m}}$ is the Lagrange multiplier associated with $C_{1}^{\prime}$, and $C_{2}^{\prime}$ is absorbed into KKT conditions. The equivalent dual problem can be decomposed as [42]

$$
\min _{\left(\alpha_{i_{m}} \geq 0\right)} \max _{\left(\tilde{\mathbf{p}}_{i_{m}}^{d}\right)} \cdot \mathcal{L}_{E E}\left(\tilde{\mathbf{p}}_{i_{m}}^{d}, \alpha_{i_{m}}\right) .
$$

The inner maximization problem solves the resource allocation problem and the outer minimization problem solves the master dual problem.
The optimal value $\hat{p}_{i_{m}}^{k}$ corresponding to $q_{i_{m}}^{d}$ is given by

$\hat{p}_{i_{m}}^{k}=\left[\frac{\eta \log _{2} e}{q_{i_{m}}^{d}+\eta \alpha_{i_{m}}}-\frac{I_{i_{m}, 1}^{d}+I_{i_{m}, 2}^{d}+I_{i_{m}, 1}^{c}+I_{i_{m}, 2}^{c}+N_{0}}{g_{i_{m}}^{k}}\right]^{+}$

where $[x]^{+}=\max \{0, x\}$. Equation (24) indicates a waterfilling algorithm, where the water level is determined by the cost of allocating resource, i.e., $\alpha_{i_{m}}$, as well as the current cost of total power consumption given by $q_{i_{m}}^{d}$. For solving the minimization problem, the Lagrange multipliers can be updated by using the gradient method [43] as

$$
\begin{aligned}
& \alpha_{i_{m}}(\tau+1) \\
& =\left[\alpha_{i_{m}}(\tau)+\mu_{i_{m}, \alpha}(\tau)\left(\sum_{k \in \mathcal{K}_{m}} \hat{p}_{i_{m}}^{k}(\tau)-p_{i_{m}, \max }^{d}\right)\right]^{+},
\end{aligned}
$$

where $\tau$ is the iteration index, $\mu_{i_{m}, \alpha}$ is the positive step size. We have adopted a constant step size to strike a balance between optimality and convergence speed. Then, $q_{i_{m}}^{d}$ is updated for the next iteration as

$$
q_{i_{m}}^{d}=\frac{C_{i_{m}}^{d}\left(\hat{\mathbf{p}}_{i_{m}}^{d}\right)}{p_{i_{m}, t}^{d}\left(\hat{\mathbf{p}}_{i_{m}}^{d}\right)}
$$

The iteration process will continue until $C_{i_{m}}^{d}\left(\hat{\mathbf{p}}_{i_{m}}^{d}\right)-$ $q_{i_{m}}^{d} p_{i_{m}, t}^{d}\left(\hat{\mathbf{p}}_{i_{m}}^{d}\right) \leq \Delta_{i_{m}}^{d}$, or the maximum iteration number is reached. $\Delta_{i_{m}}^{d}$ is the maximum tolerance. Then we set $\tilde{\mathbf{p}}_{i_{m}}^{d *}=\hat{\mathbf{p}}_{i_{m}}^{d}$, and calculate $q_{i_{m}}^{d *}$ as (16). The optimization problem (21) can be solved in a similar way, which is omitted here due to space limitation.

On the other hand, in order to obtain the optimum channel selection strategy, we can take the derivative of the suboptimal problem w.r.t. $s_{i_{m}}^{k}$, which yields $\frac{\partial \mathcal{L}_{E E}}{\partial s_{i_{m}}^{k}}$. Thus, the $k$-th channel is selected if $\frac{\partial \mathcal{L}_{E E}}{\partial s_{i m}^{k}}>0$, which provides the $i$-th $\mathrm{D} 2 \mathrm{D}$ pair with a positive marginal benefit.

The convergence of the algorithm to the maximum EE is guaranteed by the following theorem:

Theorem 2: The iterative optimization algorithm converges to the maximum $\mathrm{EE}$ which is unique if the transformed problems (20) and (21) are feasible [31], [39], [43].

\section{Nash Equilibrium Analysis}

Definition 1. A Nash equilibrium is a set of strategies that none UE (neither D2D UE nor cellular UE) can unilaterally improve its EE performance by choosing a different strategy set.

Theorem 3: The strategy set $\left\{\mathbf{s}_{i_{m}}^{d *}, \mathbf{p}_{i_{m}}^{d *}, \mathbf{s}_{k_{m}}^{c *} \mid \forall i \in\right.$ $\left.\mathcal{N}_{m}, \forall k \in \mathcal{K}_{m}, \forall m \in \mathcal{M}\right\}$, obtained by the proposed algorithm constitutes a Nash equilibrium, which exists but is not unique.

Proof: The proof of Theorem 3 is given in Appendix A. 


\section{The Centralized Interference Mitigation Algorithm}

From Theorem 2, we know that if the QoS constraint $C_{3}$ is not satisfied, (21) becomes infeasible, and the convergence of the proposed iterative algorithm cannot be guaranteed. To measure the probability of infeasibility, i.e., $\operatorname{Pr}\left\{C_{k_{m}}^{c *}<C_{k_{m}, \text { min }}^{c}\right\}$, we adopt infeasibility ratio as a key performance measurement for D2D communications [11]. Infeasibility ratio and can be quite high if RRHs are densely deployed. In this subsection, we propose an interference mitigation algorithm to reduce the infeasibility ratio by exploiting the powerful computing capabilities of the centralized BBU pool.

The functional block diagram of the proposed algorithm is shown in Fig. 3. In the first stage, the interference caused by cellular UEs is canceled by interference regeneration based techniques such as network interference cancellation engine (NICE) [44]. We target the intercell interference from cellular UEs firstly because the transmission power of cellular UEs is usually much higher than D2D UEs due to longer transmission distances between cellular UEs and RRHs. In particularly, cellular UEs near the cell edge may cause significant interference.

NICE opportunistically performs interference cancellation by exchanging decoded interferer's data within the centralized BBU pool. The suffering BBU performs channel estimation to identify dominant interferers, and requests the decoded data associated with those dominant interferers from corresponding BBUs. Since the signal processing ability is limited, an interfering signal is likely to be selected for cancellation only if its signal strength is large enough. Let $\Phi_{k_{m}}^{c}$ represent the set of dominant interferer UEs and $r_{k_{m}}^{c}$ represent the received signal on the $k$-th channel in the $m$-th cell. The interfering signal $y_{m^{\prime}}^{c}$ that is reconstructed for all $m^{\prime} \in \Phi_{k_{m}}^{c}$, is subtracted form the overall received signal as

$$
\hat{r}_{k_{m}}^{c}=r_{k_{m}}^{c}-\sum_{m^{\prime} \in \Phi_{k_{m}}^{c}} y_{m^{\prime}}^{c}
$$

This results in a new post-cancellation of the received signal vector with reduced interference level. The detailed mathematical derivations can be found in [44].

In the second stage, both the intracell interference and intercell interference caused by D2D UEs are suppressed by dynamically adjusting the maximum tolerable transmission power for each D2D pair. For example, the optimization of $p_{i_{m}, \max }^{k}$ is performed in the centralized BBU pool, and is informed to the $i$-th D2D transmitter by distributed RRHs. Then the D2D transmitter is forced to transmit at a lower power level than $p_{i_{m}, \max }^{k}$ that should not cause too much interference to cellular UEs in adjacent cells.

The constraint $C_{1}^{\prime}$ is replaced by the following $K_{m}$ constraints:

$$
C_{i_{m}, 1} \sim C_{i_{m}, K_{m}}: 0 \leq \tilde{p}_{i_{m}}^{k} \leq p_{i_{m}, \max }^{k}, \forall k \in \mathcal{K}_{m}
$$

In addition, $p_{i_{m}, \max }^{k}$ should also satisfy

$$
C_{i_{m}, K_{m}+1}: \sum_{k \in \mathcal{K}_{m}} p_{i_{m}, \max }^{k} \leq p_{i_{m}, \max }^{d} .
$$

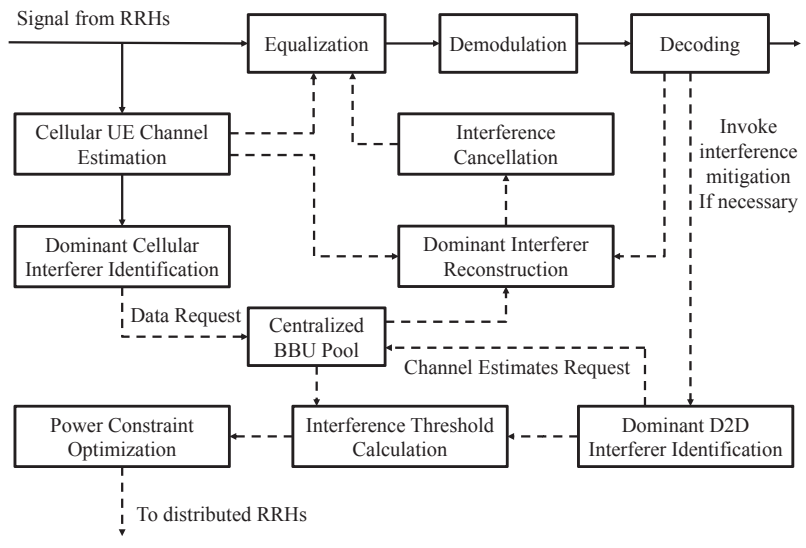

Fig. 3. Block diagram of centralized interference mitigation schemes

For a given $C_{k_{m}, \min }^{c}$ in $C_{3}$, the interference threshold for the $k$-th cellular UE in the $m$-th cell is calculated as

$$
I_{k_{m}, 1}^{d}+I_{k_{m}, 2}^{d} \leq \frac{p_{k_{m}}^{c} g_{k_{m}}^{c}}{2^{C_{k_{m}, m i n}^{c}-1}}-N_{0}=I_{k_{m}, t h}^{c} .
$$

The above interference threshold can be rewritten as

$$
\begin{aligned}
C_{k_{m}, 1} & : \sum_{i \in \mathcal{N}_{m}} p_{i_{m}, \max }^{k} g_{i_{m}, k_{m}} \\
& +\sum_{m^{\prime} \in \mathcal{M} \backslash\{m\}} \sum_{j_{m^{\prime}} \in N_{m^{\prime}}} p_{j_{m^{\prime}}, \max }^{k} g_{j_{m^{\prime}}, k_{m}} \leq I_{k_{m}, t h}^{c} .
\end{aligned}
$$

Define $\mathbf{p}_{\max }^{d}=\left\{p_{i_{m}, \max }^{k} \mid \sum_{k \in \mathcal{K}_{m}} p_{i_{m}, \max }^{k} \leq p_{i_{m}, \max }^{d}\right\}$, $\forall m \in \mathcal{M}, \forall k \in \mathcal{K}_{m}, \forall i \in \mathcal{N}_{m}$, which can be obtained by solving the following problem

$$
\begin{aligned}
& \max _{\mathbf{p}_{\text {max }}^{d}} \prod_{m \in \mathcal{M}} \prod_{i \in \mathcal{N}_{m}} \prod_{k \in \mathcal{K}_{m}}\left(\frac{p_{i_{m}, \max }^{k}}{w_{i_{m}}^{k}}\right) \\
& \text { s.t. } \quad C_{i_{m}, K_{m}+1}, C_{k_{m}, 1}, \forall m \in \mathcal{M}, \forall k \in \mathcal{K}_{m}, \forall i \in \mathcal{N}_{m} .
\end{aligned}
$$

$w_{i_{m}}^{k}$ is the weight of $p_{i_{m}, \max }^{k}$. If $w_{i_{m}}^{k}=1, \forall m \in \mathcal{M}, \forall k \in$ $\mathcal{K}_{m}, \forall i \in \mathcal{N}_{m}$, the RRH allocates resources based on the instantaneous interfering channel gains. D2D UEs that would cause stronger interference are always allocated with less resources, which may be unfair in the long run. Alternatively, $w_{i_{m}}^{k}$ can be designed to take fairness into consideration such as the proportional fair scheduling [36].

It is noted that if the D2D interference mitigation scheme is used, the constraint $C_{1}^{\prime}$ should be replaced by $C_{i_{m}, 1} \sim$ $C_{i_{m}, K_{m}}$, which are even tighter constraints. The optimal value $\hat{p}_{i_{m}}^{k}$ is similar to (24) by replacing $\alpha_{i_{m}}$ with $\beta_{i_{m}}^{k}$, which is the Lagrange multiplier associated with $C_{i_{m}, k}$. Then, $\beta_{i_{m}}^{k}$ is updated as

$$
\beta_{i_{m}}^{k}(\tau+1)=\left[\beta_{i_{m}}^{k}(\tau)+\mu_{i_{m}, \beta}^{k}(\tau)\left(\hat{p}_{i_{m}}^{k}(\tau)-p_{i_{m}, \max }^{k}\right)\right]^{+}
$$

The impacts of using tighter constraints $C_{i_{m}, 1} \sim C_{i_{m}, K_{m}}$ instead of $C_{1}^{\prime}$ are analyzed through simulation results. 


\section{E. Implementation Issues and Complexity Analysis}

When implementing the algorithm, channel estimation is particularly important since the efficiency of both interference mitigation and resource allocation depends on channel estimation accuracy. In conventional cellular systems, channel estimation is performed at the base station (BS) side, and the BS has to be aware of the reference symbols assigned by neighboring BSs. In addition, the decoded data have to be firstly decoded by interfering BSs and then transmitted back to the desired BS through backhaul links. The cooperation among cells causes frequent signaling exchange, and may not perform well due to backhaul delay, synchronization errors, etc. However, in the novel C-RAN architecture, information exchange and optimization can be performed in the centralized BBU pool, which facilitates the implementation of the proposed algorithm. In this paper, we assume that precise channel estimates are available. The impacts of channel estimation errors are discussed through simulation results.

In the distributed resource allocation algorithm, each D2D pair only needs to estimate the received interference rather than knowing the specific power allocation or channel selection strategies of interferers. The reason is that the sufficient information of strategies are contained in the form of interference. Cellular UEs also need to know the knowledge of interference, which can be estimated firstly by centralized powerful BBUs and then fed back to cellular UEs through distributed RRHs.

The iterative algorithm produces an increasing sequence of $q_{i_{m}}^{d}$ or $q_{k_{m}}^{c}$ values, which converge to the optimum value $q_{i_{m}}^{d *}$ or $q_{k_{m}}^{c *}$ at a super-linear convergence rate [43]. Taking (20) as an example, the complexity is dominated by the calculations given by (24), which leads to a total complexity $\mathcal{O}\left(K_{m} L_{i_{m}, 1}^{d} L_{i_{m}, 2}^{d}\right)$, where $L_{i_{m}, 1}^{d}$ and $L_{i_{m}, 2}^{d}$ are the numbers of iterations required for reaching convergence and solving the dual problem respectively.

The overhead caused by the interference mitigation algorithm consists of two parts, i.e., the regeneration of intercell interference caused by cellular UEs, and the optimization of the maximum transmission power cap for D2D pairs. In the first part, the interference cancellation algorithm is, in essence, a "multi-cell successive interference cancellation" (MC-SIC) technique with a low computation complexity that is of the same order as conventional SIC receivers. The interference cancellation algorithm is invoked only if the intercell interference exceeds a certain threshold, and only interference with large enough signal strength will be selected for signal reconstruction. According to [44], the overhead factor is only 1.9 when there are six dominant interference sources, which causes almost two orders of magnitude less overheads than multi-antenna based schemes. In the second part, the optimization problem defined in (32) is a geometric programming problem, and can be transformed to a convex problem by a change of variables and a transformation of the objective and constraint functions. It involves solving a convex problem with $\sum_{m \in \mathcal{M}} N_{m} K_{m}$ optimization parameters, and $\sum_{m \in \mathcal{M}}\left(N_{m}+K_{m}\right)$ linear inequality constraints, which can be solved in polynomial time. If D2D UEs in the same cell or clusters are allocated with the same resource, i.e.,
TABLE II. SIMUlation PARAMETERS.

\begin{tabular}{|l|l|}
\hline Parameter & Value \\
\hline Cell radius & $300 \mathrm{~m}$ \\
\hline Intercell distance & $500 \mathrm{~m}$ \\
\hline Max D2D transmission distance & $25 \mathrm{~m}$ \\
\hline Max Tx power $p_{i_{m}, \max }^{d}, p_{k_{m}, \max }^{c}$ & $200 \mathrm{~mW}(23 \mathrm{dBm})$ \\
\hline Constant circuit power $p_{c i r}$ & $10 \mathrm{~mW}(10 \mathrm{dBm})$ \\
\hline Thermal noise power $N_{0}$ & $10^{-7} \mathrm{~W}$ \\
\hline Number of D2D pairs $N_{m}$ & 16 \\
\hline Number of cellular UEs $K_{m}$ & 16 \\
\hline PA efficiency $\eta$ & $35 \%$ \\
\hline QoS of cellular UEs $C_{k_{m}, \min }^{c}$ & $0 \sim 1 \mathrm{bit} / \mathrm{s} / \mathrm{Hz}$ \\
\hline Number of RRHs $M$ & 7 \\
\hline
\end{tabular}

$p_{i_{m}, \max }^{k}=p_{j_{m, \max }}^{k}, \forall m \in \mathcal{M}, \forall i, j \in \mathcal{N}_{m}, i \neq j$, the number of optimization parameters and inequality constraints are further reduced to $\sum_{m \in \mathcal{M}} K_{m}$ and $\sum_{m \in \mathcal{M}}\left(K_{m}+1\right)$, respectively. As a result, current fronthaul technologies are indeed able to provide enough capacity to implement the proposed interference cancellation and mitigation algorithm, which provides an flexible tradeoff between energy efficiency improvements and fronthaul overhead.

\section{Simulation Results}

In this section, the proposed hybrid energy-efficient algorithm, labeled as "hybrid scheme", is compared with previous works [31], [32], which are labeled as "conventional scheme". The values of simulation parameters are based on [9], [27], [28], [31], and are summarized in Table II. The maximum transmission power of cellular UEs is the same as D2D UEs, i.e., $p_{k_{m}, \max }^{c}=p_{i_{m}, \max }^{d}=200 \mathrm{~mW}(23 \mathrm{dBm})$. The channel gain between the transmitter $i$ and the receiver $j$ is calculated as $d_{i, j}^{-2}\left|h_{i, j}\right|^{2}$, where $d_{i, j}$ is the distance between the transmitter $i$ and the receiver $j, h_{i, j}$ is the complex Gaussian channel coefficient that satisfies $h_{i, j} \sim \mathcal{C N}(0,1)$. The simulation results are averaged through a total number of $10^{3}$ simulations. The locations of cellular UEs and D2D UEs are generated randomly in each simulation.

Fig. 4 shows the infeasibility ratio corresponding to various QoS requirements. The minimum QoS requirement of cellular UEs $C_{k_{m}, \text { min }}^{c}$ is increased from 0 to $1 \mathrm{bits} / \mathrm{s} / \mathrm{Hz}$ with a step of 0.1 , and the corresponding infeasibility ratio is obtained through simulations. We observe that as the QoS requirement increases, the infeasibility ratio of the conventional algorithm increases dramatically due to the severe interference. In comparison, the proposed hybrid scheme achieves a nearly "zero" infeasibility ratio.

Fig. 5 shows the average channel selection error rate versus the normalized mean square error (NMSE) of channel estimates. A channel selection error is defined that the channel should have been selected but was not, or otherwise. The total number of errors is divided by the total number of selections, and averaged over $10^{5}$ simulations. NMSE is defined as [45]

$$
\mathrm{NMSE}=\frac{1}{N} \sum_{n=1}^{N}\left|\frac{\hat{h}_{i, j}-h_{i, j}}{h_{i, j}}\right|^{2},
$$




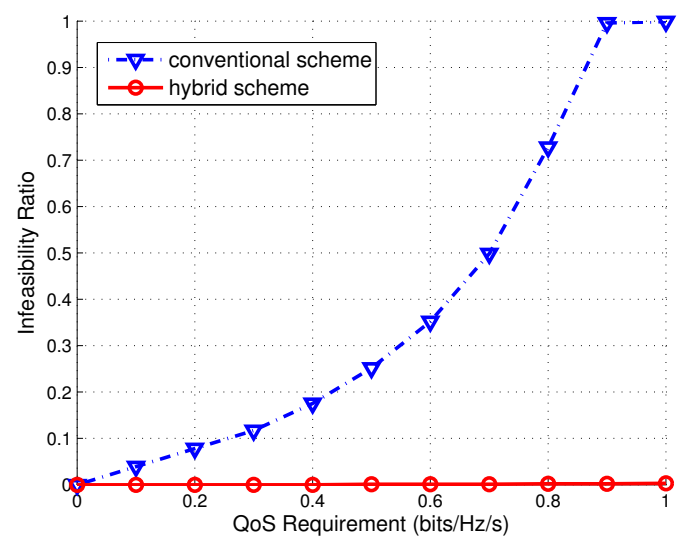

Fig. 4. The infeasibility ratio versus QoS requirements $\left(N_{m}=K_{m}=16\right.$, $\left.m=1, \cdots, 7, C_{k_{m}, \min }^{c}=0 \sim 1 \mathrm{bits} / \mathrm{s} / \mathrm{Hz}\right)$.

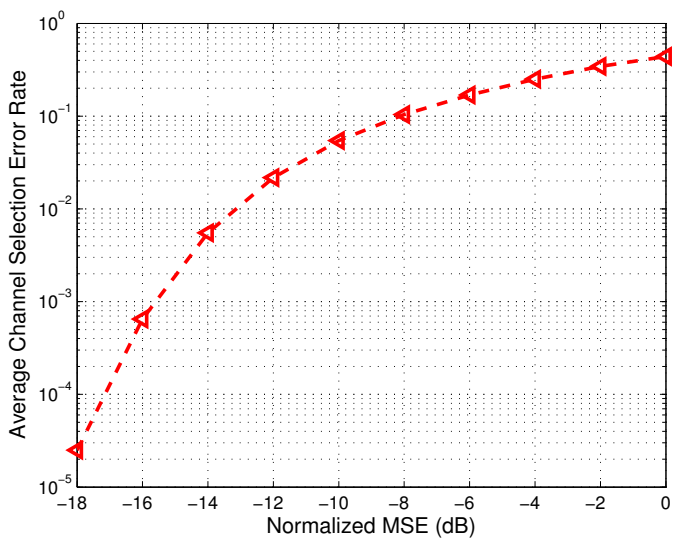

Fig. 5. The average channel selection error rate versus the normalized mean square error (NMSE) of channel estimates. $\left(N_{m}=K_{m}=16\right.$, $\left.m=1, \cdots, 7, C_{k_{m}, \min }^{c}=0.7 \mathrm{bits} / \mathrm{s} / \mathrm{Hz}\right)$.

where $\hat{h}_{i, j}$ is the estimate of $h_{i, j}$. As shown in Fig. 5, the proposed algorithm is robust to small estimation errors, e.g., it achieves an average channel selection error rate of $10^{-2}$ when NMSE $=-14 \mathrm{~dB}$. As the NMSE increases, the average channel selection error rate continually increases to 0.5 .

Fig. 6 and Fig. 7 show the average EE of cellular UEs and D2D UEs corresponding to the number of game iterations respectively. We assume that both cellular and D2D UEs are forced to stop transmission and reschedule resource if the QoS requirement cannot be satisfied due to interference. In this way, we have taken infeasible scenarios into considerations and avoided only using good channel conditions of feasible scenarios for comparison. Fig. 6 shows that the hybrid scheme improves the EE performance by $91 \%$ and $300 \%$ for $C_{k_{m}, \min }^{c}=0.2$ and $C_{k_{m}, \min }^{c}=0.7$ respectively. The improvement continues to increase as the QoS requirement increases. The reason is that as the QoS requirement increases,

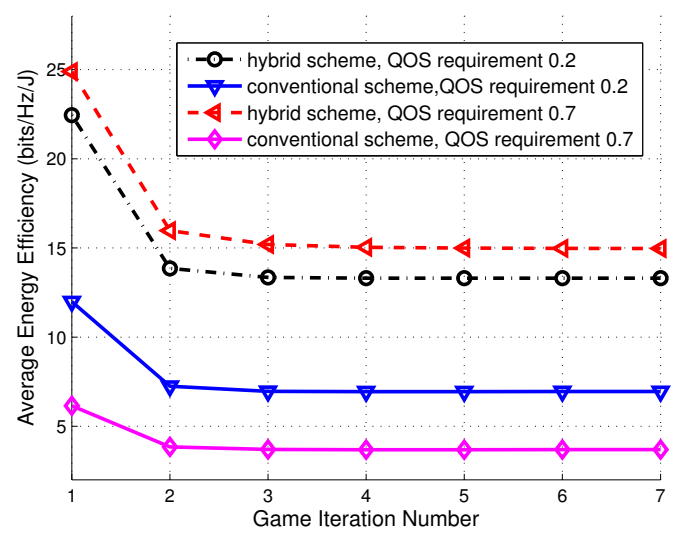

Fig. 6. The average energy efficiency of cellular UEs corresponding to the number of game iterations $\left(N_{m}=K_{m}=16, m=1, \cdots, 7, C_{k_{m}, \min }^{c}=\right.$ $0.2,0.7 \mathrm{bits} / \mathrm{s} / \mathrm{Hz})$.

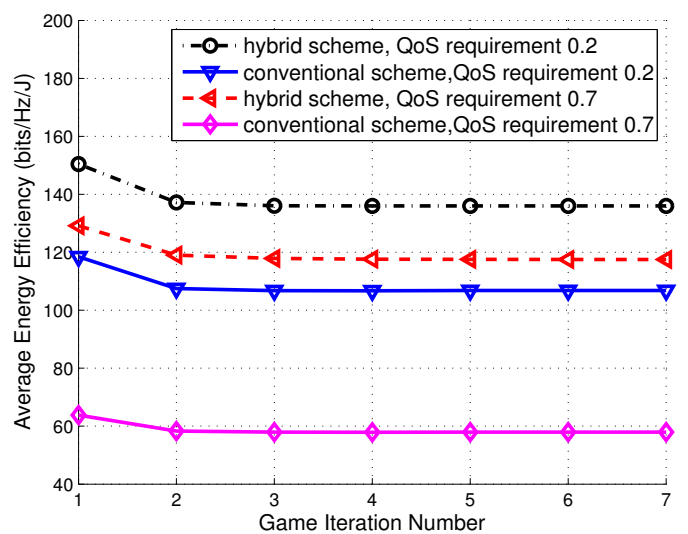

Fig. 7. The average energy efficiency of D2D UEs corresponding to the number of game iterations $\left(N_{m}=K_{m}=16, m=1, \cdots, 7, C_{k_{m}, \text { min }}^{c}=\right.$ $0.2,0.7 \mathrm{bits} / \mathrm{s} / \mathrm{Hz})$.

the infeasibility ratio also increases and both D2D and cellular UEs have to wait a much longer time for rescheduling resource allocation.

Fig. 7 shows an amazing result that D2D UEs can also benefit from interference mitigation. The hybrid scheme improves the EE performance by $27 \%$ and $102 \%$ for $C_{k}^{c}$ min $=0.2$ and $C_{k_{m}, \min }^{c}=0.7$ respectively. The reasons that D2D UEs can also improve EE performance even with more strict transmission power constraints are two folds: first, the constraint reduces the interference caused to cellular UEs and thus requires less transmission power from cellular UEs. Since the transmission power of cellular UEs is usually much higher than D2D UEs due to longer transmission distances between cellular UEs and RRHs, this transmission power reduction in turn reduces the severe interference to D2D UEs and improves the EE performance; second, it reduces the infeasibility ratio dramatically, which in turn reduces the idle time of D2D UEs 
and improves the EE performance since both cellular and D2D UEs are forced to stop transmission and wait for resource reallocation when the QoS requirement cannot be satisfied. However, we also observe that the improvement for D2D UEs is less than that of cellular UEs since cellular UE has a higher priority and strict QoS requirements. In addition, both Fig. 6 and Fig. 7 also demonstrate that the proposed algorithm converges efficiently to the optimum strategy within $3 \sim 4$ iterations.

\section{CONCLUSION}

In this paper, we proposed a hybrid resource allocation algorithm for D2D communications underlaying C-RAN based LTE-A networks. The proposed algorithm consists of a centralized interference mitigation algorithm that is performed in the centralized BBU pool, and a distributed joint channel selection and power allocation algorithm that is performed by individual UEs with the aid of distributed RRHs. Simulation results show that the proposed scheme achieves a nearly "zero" infeasibility ratio, and improves the EE performance by $300 \%$ for cellular UEs and $102 \%$ for D2D UEs with a QoS requirement of 0.7 bits $/ \mathrm{s} / \mathrm{Hz}$. The results validate the effectiveness of the proposed algorithm.

\section{APPENDIX A}

\section{PROOF OF THE THEOREM 3}

According to [46], a Nash equilibrium exists if the utility function is continuous and quasiconcave, and the set of strategies is a nonempty compact convex subset of a Euclidean space. Taking the EE objection function defined in (12) as an example, after the relaxation of $s_{i_{m}}^{k}$ from a Boolean value to a real value, the numerator $C_{i_{m}}^{d}$ defined in (1) is a concave function of $s_{i_{m}}^{k} p_{i_{m}}^{k}, \forall i \in \mathcal{N}, k \in \mathcal{K}, m \in \mathcal{M}$. The denominator defined in (10) is an affine function of $s_{i_{m}}^{k} p_{i_{m}}^{k}$. Therefore, $E_{i_{m}}^{d}$ is quasiconcave (Problem 4.7 in [42]). The set of the strategies $\left\{\mathbf{s}_{i_{m}}^{d}, \mathbf{p}_{i_{m}}^{d}\right\}$ is a nonempty compact convex subset of the Euclidean space. Similarly, it is easily proved that the above conditions also hold for the cellular UE. Therefore, a Nash equilibrium exists in the noncooperative game.

If the strategy set $\left\{\mathbf{s}_{i_{m}}^{d *}, \mathbf{p}_{i_{m}}^{d *}\right\}$ obtained by the iterative algorithm is not the Nash equilibrium, the D2D transmitter can choose the Nash equilibrium $\left\{\hat{\mathbf{s}}_{i_{m}}^{d}, \hat{\mathbf{p}}_{i_{m}}^{d}\right\}\left(\left\{\hat{\mathbf{s}}_{i_{m}}^{d}, \hat{\mathbf{p}}_{i_{m}}^{d *}\right\} \neq\right.$ $\left.\left\{\mathbf{s}_{i_{m}}^{d *}, \mathbf{p}_{i_{m}}^{d *}\right\}\right)$ to obtain the maximum EE $q_{i_{m}}^{d *}$. However, by Theorem 1 and Theorem 2, $q_{i_{m}}^{d *}$ can also be achieved by choosing $\left\{\mathbf{s}_{i_{m}}^{d *}, \mathbf{p}_{i_{m}}^{d *}\right\}$, and $q_{i_{m}}^{d *}$ is unique. As a result, $\left\{\mathbf{s}_{i_{m}}^{d *}, \mathbf{p}_{i_{m}}^{d *}\right\}$ is also part of a Nash equilibrium. A similar proof holds for $\left\{\mathbf{p}_{k_{m}}^{d *}\right\}$.

However, despite that there may be multiple equilibria, the maximum EE obtained by the iterative algorithm is unique. The proof is similar to the proof of Lemma 4 in [39] of the resubmitted manuscript, which proves that the optimum result obtained by nonlinear fractional programming is unique. This completes the proof.

\section{REFERENCES}

[1] R. Wang, H. Hu, and X. Yang, "Potentials and challenges of C-RAN supporting multi-RATs toward 5G mobile networks," IEEE Access., vol. 2, no. 8, pp. 1187-1194, Oct. 2014.
[2] O. Bello and S. Zeadally, "Intelligent device-to-device communications in the internet of things," IEEE Sys. J., vol. PP, no. 99, pp. 1-11, Jan. 2014.

[3] K. Doppler, M. Rinne, C. Wijting, C. B. Ribeiro et al., "Device-todevice communication as an underlay to LTE-Advanced networks," IEEE Comm. Mag., vol. 47, no. 12, pp. 42-49, Dec. 2009.

[4] K. Zheng, F. Hu, W. Wang, W. Xiang, and M. Dohler, "Radio resource allocation in LTE-Advanced cellular networks with M2M communications," IEEE Commun. Mag., vol. 50, no. 7, pp. 184-192, Aug. 2012.

[5] G. Rigazzi, N. K. Pratas, P. Popovski, and R. Fantacci, "Aggregation and trunking of M2M traffic via D2D connections," in Proc. IEEE ICC'15, London, UK, Jun. 2015, pp. 1-6.

[6] M. N. Tehrani, M. Uysal, and H. Yanikomeroglu, "Device-to-device communications in 5G cellular networks: challenges, solutions, and future directions," IEEE Comm. Mag., vol. 52, no. 5, pp. 86-92, May. 2014

[7] J. Liu, Y. Kawamoto, H. Nishiyama, N. Kato, and N. Kadowaki, "Device-to-device communications achieve efficient load balancing in LTE-Advanced networks," IEEE Wirel. Comm. Mag., vol. 21, no. 2, pp. 57-65, Apr. 2014.

[8] X. Lin, J. Andrews, A. Ghosh, and R. Ratasuk, "An overview of 3GPP device-to-device proximity services," IEEE Comm. Mag., vol. 52, no. 4, pp. 40-48, Apr. 2014.

[9] S. Luo, R. Zhang, and T. J. Lim, "Downlink and uplink energy minimization through user association and beamforming in C-RAN," IEEE Trans. Wirel. Comm., vol. 14, no. 1, pp. 494-508, Aug. 2014.

[10] A. Davydov, G. Morozov, I. Bolotin, and A. Papathanassiou, "Evaluation of joint transmission CoMP in C-RAN based LTE-A HetNets with large coordination areas," in Proc. IEEE GLOBECOM'13, Atlanta, GA, USA, Dec. 2013, pp. 801-806.

[11] G. Fodor, E. Dahlman, G. Mildh, S. Parkvall et al., "Design aspects of network assisted device-to-device communications," IEEE Comm. Mag., vol. 50, no. 3, pp. 170-177, Mar. 2012.

[12] M. Jo, T. Maksymyuk, B. Strykhalyuk, and C. H. Cho, "Deviceto-device-based heterogeneous radio access network architecture for mobile cloud computing," IEEE Wirel. Comm. Mag., vol. 22, no. 3, pp. 50-58, Jun. 2015.

[13] R. Yin, G. Yu, C. Zhong, and Z. Zhang, "Distributed resource allocation for D2D communication underlaying cellular networks," in Proc. IEEE ICC'13, Budapest, Hungary, Jun. 2013, pp. 138-143.

[14] F. Wang, L. Song, Z. Han, Q. Zhao et al., "Joint scheduling and resource allocation for device-to-device underlay communication," in Proc. IEEE WCNC'13, Shanghai, China, Apr. 2013, pp. 134-139.

[15] D. Lee, K. Choi, W. Jeon, and D. Jeong, "Two-stage semi-distributed resource management for device-to-device communication in cellular networks," IEEE Trans. Wirel. Comm., vol. PP, no. 99, pp. 1-13, Feb. 2014

[16] D. Feng, L. Lu, Y. Yi, G. Y. Li et al., "Device-to-device communications underlaying cellular networks," IEEE Trans. Comm., vol. 61, no. 8, pp. 3541-3551, Aug. 2013.

[17] C. Xu, L. Song, Z. Han, and Q. Zhao, "Efficiency resource allocation for device-to-device underlay communication systems: a reverse iterative combinatorial auction based approach,' IEEE J. Sel. Areas Commun., vol. 31, no. 9, pp. 348-358, Sep. 2013.

[18] M. Hasan, E. Hossain, and D. Kim, "Resource allocation under channel uncertainties for relay-aided device-to-device communication underlaying LTE-A cellular networks," IEEE Trans. Wirel. Comm., vol. PP, no. 99, pp. 1-17, Mar. 2014.

[19] B. Zhou, H. Hu, S. Huang, and H. Chen, "Intracluster device-to-device relay algorithm with optimal resource utilization," IEEE Trans. Veh. Tech., vol. 62, no. 5, pp. 2315-2326, Jun. 2013.

[20] Y. Cheng, Y. Gu, and X. Lin, "Combined power control and link selection in device-to-device enabled cellular systems," IET Commun., vol. 7, no. 12, pp. 1221-1230, Aug. 2013. 
[21] Y. Chia, K. Doppler, C. B. Ribeiro, and O. Tirkkonen, "Resource sharing optimization for device-to-device communication underlaying cellular networks," IEEE Trans. Wirel. Comm., vol. 10, no. 8, pp. 2752-2763, Aug. 2011.

[22] N. Golrezaei, P. Mansourifard, A. F. Molisch, and A. G. Dimakis, "Base-station assisted device-to-device communications for high-throughput wireless video networks," IEEE Trans. Wirel. Comm., vol. 13, no. 7, pp. 3665-3676, Jul. 2014.

[23] X. Zhang, Z. Zheng, Q. Shen, J. Liu, X. S. Shen, and L. L. Xie, "Optimizing network sustainability and efficiency in green cellular networks," IEEE Trans. Wirel. Comm., vol. 13, no. 2, pp. 1129-1139, Feb. 2014.

[24] C. Xu, L. Song, and Z. Han, Resource Management for Deviceto-Device Underlay Communication. Springer Briefs in Computer Science, 2014, pp. 1-79.

[25] A. Mukherjee and A. Hottinen, "Energy-efficient device-to-device MIMO underlay network with interference constraints," in Proc. IEEE WSA'12, Dresden, Germany, Mar. 2012, pp. 105-109.

[26] S. Wen, X. Zhu, Z. Lin, X. Zhang, and D. Yang, "Energy efficient power allocation schemes for device-to-device (D2D) communication," in Proc. IEEE VTC Fall'13, Las Vegas, USA, Sep. 2013, pp. 1-5.

[27] F. Wang, C. Xu, L. Song, Z. Han, and B. Zhang, "Energy-efficient radio resource and power allocation for device-to-device communication underlaying cellular networks," in Proc. IEEE WCSP'12, Huangshan, China, Oct. 2012, pp. 1-6.

[28] F. Wang, C. Xu, L. Song, Q. Zhao et al., "Energy-aware resource allocation for device-to-device underlay communication," in Proc. IEEE ICC'13, Budapest, Hungary, Jun. 2013, pp. 6076-6080.

[29] T. Ta, J. S. Baras, and C. Zhu, "Improving smartphone battery life utilizing device to device cooperative relays underlaying LTE networks," in Proc. IEEE ICC'14, Sydney, Australia, Jun. 2014, pp. 1-6.

[30] S. Mumtaz, K. M. S. Huq, A. Radwan, J. Rodriguez, and R. L. Aguiar, "Energy-efficient interference-aware resource allocation in LTE-D2D communication,' in Proc. IEEE ICC'14, Sydney, Australia, Jun. 2014, pp. $1-6$.

[31] Z. Zhou, M. Dong, K. Ota et al., "A game-theoretic approach to energyefficient resource allocation in device-to-device underlay communications," IET Commun., vol. 9, no. 3, pp. 375-385, Feb. 2015.

[32] Z. Zhou, M. Dong, K. Ota, J. Wu, and T. Sato, "Energy efficiency and spectral efficiency tradeoff in device-to-device D2D communications," IEEE Wirel. Commun. Lett., vol. 3, no. 5, pp. 485-488, Jul. 2014.

[33] D. Wu, J. Wang, R. Q. Hu, Y. Cai et al., "Energy-efficient resource sharing for mobile device-to-device multimedia communications," IEEE Trans. Veh. Tech., vol. 63, no. 5, pp. 2093-2103, Mar. 2014.

[34] H. Chen, D. Wu, and Y. Cai, "Coalition formation game for green resource management in D2D communications," IEEE Commun. Lett., vol. 18, no. 8, pp. 1395-1398, Aug. 2014.

[35] K. A. Meerja, A. Shami, and A. Refaey, "Hailing cloud empowered radio access networks," IEEE Wirel. Comm. Mag., vol. 22, no. 1, pp. 122-129, Feb. 2015.

[36] A. Goldsmith, Wireless Communications. Cambridge, UK: Cambridge University Press, 2005.

[37] Z. Han, D. Niyato, W. Saad, T. Basar, and A. Hjorungnes, Game Theory in Wireless and Communication Networks: Theory, Models, and Applications, 1st ed. Cambridge, UK: Cambridge University Press, 2012.

[38] H. Kwon and T. Birdsall, "Channel capacity in bits per joule," IEEE J. Ocean. Eng., vol. OE-11, no. 1, pp. 97-99, Jan. 1986.

[39] W. Dinkelbach, "On nonlinear fractional programming," Management Science, vol. 13, no. 7, pp. 492-498, Mar. 1967.

[40] C. Y. Wong, R. S. Cheng, K. B. Letaief, and R. D. Murch, "Multiuser OFDM with adaptive subcarrier, bit, and power allocation," IEEE J. Sel. Areas Commun., vol. 17, no. 2, pp. 1747-1758, Oct. 1999.

[41] Z. Chang, T. Ristaniemi, and Z. Niu, "Radio resource allocation for collaborative OFDMA relay networks with imperfect channel state information," IEEE Trans. Wirel. Commun., vol. 13, no. 5, pp. 28242835, May. 2014.

[42] S. Boyd and L. Vandenberghe, Convex Optimization. Cambridge, UK: Cambridge University Press, 2004.

[43] K. T. K. Cheung, S. Yang, and L. Hanzo, "Achieving maximum energy-efficiency in multi-relay OFDMA cellular networks: a fractional programming approach," IEEE Trans. Comm., vol. 61, no. 8, pp. 27462757, Jul. 2013.

[44] K. Balachandran, J. H. Kang, K. Karakayali, and K. M. Rege, "NICE: a network interference cancellation engine for opportunistic uplink cooperation in wireless networks," IEEE Trans. Wirel. Comm., vol. 10, no. 2, pp. 540-549, Feb. 2011

[45] S. Haykin, Adaptive Filter Theory, 4th ed. Upper Saddle River, USA: Prentice Hall PTR, 2001, pp. 203-227.

[46] M. J. Osborne and A. Rubinstein, A Course in Game Theory. Cambridge, MA, USA: MIT Press, 1994. 